\title{
SMALL OSCILLATIONS AND THE HEISENBERG LIE ALGEBRA
}

\author{
GABRIELA OVANDO
}

\begin{abstract}
The Adler Kostant Symes [A-K-S] scheme is used to describe mechanical systems for quadratic Hamiltonians of $\mathbb{R}^{2 n}$ on coadjoint orbits of the Heisenberg Lie group. The coadjoint orbits are realized in a solvable Lie algebra $\mathfrak{g}$ that admits an adinvariant metric. Its quadratic induces the Hamiltonian on the orbits, whose Hamiltonian system is equivalent to that one on $\mathbb{R}^{2 n}$. This system is a Lax pair equation whose solution can be computed with help of the Adjoint representation. For a certain class of functions, the Poisson commutativity on the coadjoint orbits in $\mathfrak{g}$ is related to the commutativity of a family of derivations of the $2 \mathrm{n}+1$-dimensional Heisenberg Lie algebra $\mathfrak{h}_{n}$. Therefore the complete integrability is related to the existence of an n-dimensional abelian subalgebra of certain derivations in $\mathfrak{h}_{n}$. For instance, the motion of $\mathrm{n}$-uncoupled harmonic oscillators near an equilibrium position can be described with this setting.
\end{abstract}

\section{INTRODUCTION}

A quadratic Hamiltonian is a function of the form

$$
H(x)=\frac{1}{2}(A x, x)
$$

where $x=\left(q_{1}, \ldots, q_{n}, p_{1}, \ldots, p_{n}\right)$ is a vector in $\mathbb{R}^{2 n}$ written in a symplectic basis and $A$ is a symmetric linear operator with respect to the canonical inner product (,). The Hamiltonian equation have the form

$$
x^{\prime}=J A x, \quad \text { where } J=\left(\begin{array}{cc}
0 & -I d \\
I d & 0
\end{array}\right)
$$

where $I d$ denotes the identity.

In particular the motion of $\mathrm{n}$ uncoupled harmonic oscillators can be approximated by a quadratic Hamiltonian $H$ as in (11) with $A=I d$, explicitely in coordinates $H=\frac{1}{2} \sum_{i}\left(p_{i}^{2}+\right.$ $\left.q_{i}^{2}\right)$ where $q_{i}$ denote the position coordinates and $p_{i}=\dot{q}_{i}$ are the canonical momentum coordinates. Then (2) yields the equations of motion, which predict the position and the velocity at any time if initial conditions $q_{i}\left(t_{0}\right), p_{i}\left(t_{0}\right)=\dot{q}_{i}\left(t_{0}\right)$ are known.

In quantum mechanics a good approach to the simple harmonic oscillator is through the Heisenberg Lie algebra. In dimension three this is the Lie algebra generated by the position operator $Q=$ multiplication by $x$, the momentum operator $P=-i \frac{d}{d x}$ and 1 with the only non trivial commutation relation

$$
[Q, P]=1
$$

These operators evolve according to the Heisenberg equations

$$
\frac{d P}{d t}=-Q \quad \frac{d Q}{d t}=P
$$

In this work we shall show that the $2 n+1$-dimensional Heisenberg Lie algebra $\mathfrak{h}_{n}$ allows an approach to systems associated to quadratic Hamiltonians of the form (11) in classical

(2000) Mathematics Subject Classification: 70G65, 70H05, 70H06, 22E70, 22E25 .

Key words and phrases: Small oscillations, Hamiltonian systems, coadjoint orbits, Heisenberg Lie group, solvable Lie group . 
mechanics. To this end we shall make use of Lie theory. Lie theory was successful when studying some mechanical systems such as the rigid body or the generalized Toda lattice A] Ko2 Sy [R2]. In this setting ad-invariant functions play an important role. On the one hand their Hamiltonian systems becomes a Lax equation and on the other hand they are in involution on the orbits. Whenever studying Poisson commuting conditions the ad-invariance property can be replaced by a weaker one as in R1. In the framework of this theory what we need is a Lie algebra with an ad-invariant metric, a splitting of this Lie algebra into a direct sum as vector subspaces of two subalgebras and a given function. These algebraic tools were used with semisimple Lie algebras, where the Killing form is the natural candidate for the ad-invariant metric.

However there are more Lie algebras admitting an ad-invariant metric. For our purposes we are interested in the solvable ones. They can be constructed by a double extension procedure, whose more simple application follows from $\mathbb{R}^{m}$. We get a solvable Lie algebra $\mathfrak{g}$, that results a semidirect extension of the $2 \mathrm{n}+1$-dimensional Heisenberg Lie algebra $\mathfrak{h}_{n}$ and that can be endowed with an ad-invariant metric which is an extension of the bilinear form on $\mathbb{R}^{2 n}$ given by $b(x, y)=(A x, y)$ if $($,$) is the canonical inner product on \mathbb{R}^{2 n}$ and $A$ is a non singular symmetric transformation. The set of symmetric maps $A$ is in a bijective correspondence with the derivations of the Heisenberg Lie algebra acting trivially on the center, a set denoted with $\mathfrak{d}$. Any such derivation allows a semidirect extension $\mathfrak{g}$ that admits an ad-invariant metric.

The Lie algebra $\mathfrak{g}$ splits naturally as a direct sum of vector spaces of a one dimensional Lie algebra and the Heisenberg ideal. Looking the coadjoint orbits of the Heisenberg Lie group on $\mathfrak{g}$ via the metric, one gets Hamiltonian systems on these orbits. In particular for the restriction of the quadratic corresponding to the ad-invariant metric we obtain a Hamiltonian system equivalent to (11). Since the considered function is ad-invariant the system becomes a Lax equation, whose solution can be computed with the Adjoint representation.

As example we work out the linear equation of motion of n-uncoupled harmonic oscillators. The Lie algebra $\mathfrak{g}$ is known as a oscillator Lie algebra and the corresponding ad-invariant metric is Lorentzian. This Lie algebra belongs to the exclusive class of Lie algebras admitting an ad-invariant Lorentzian metric. The quadratic for this metric induces the Hamiltonian system on the orbits, whose solutions are bounded. Furthermore it is proved that the Hamiltonian is completely integrable on all maximal orbits. We notice that the functions in involution we are making use, are not ad-invariant and they do not satisfy the involution conditions of [R1].

Going back to the general case we study involution conditions on the orbits for a class of quadratic functions which are not ad-invariant in $\mathfrak{g}$ in general. They are functions of the form (11) extended to $\mathfrak{g}$. The Poisson commutativity conditions we get for these functions can be read off in the Lie algebra of derivations of $\mathfrak{h}_{n}$. If the centralizer of $J A$ in $\mathfrak{d}(\mathrm{J}$ as in (2) and $A$ as above) contains an n-dimensional abelian subalgebra, then the function (11) is completely integrable on $\mathbb{R}^{2 n}$ whose related one on $\mathfrak{g}$ is completely integrable on all orbits. This reduces the complete integrability of these systems to algebraic conditions on $s p(n)$, the Lie algebra of derivations of $\mathfrak{h}_{n}$ acting trivially on the center. Applying results of Lie Theory, one can see that many of these Hamiltonians are completely integrable. One needs to study the abelian subalgebras of $s p(n)$. In particular for the case of the motion of n-uncoupled harmonic oscillators we need a abelian subalgebra in the Lie algebra of isometries of the Heisenberg Lie group $\mathbb{H}_{n}$, endowed with its canonical inner product. 


\section{Preliminaries}

Let $G$ be a Lie group with Lie algebra $\mathfrak{g}$ and exponential map exp $: \mathfrak{g} \rightarrow G$.

Let us introduce a kind of Lie algebras we shall work with. For each integer $i \geq 1$ define $\mathfrak{g}^{i}=\left[\mathfrak{g}, \mathfrak{g}^{i-1}\right]$, where $\mathfrak{g}^{0}=\mathfrak{g}$. The Lie algebra $\mathfrak{g}$ is nilpotent if $\mathfrak{g}^{i}=0$ for some positive integer $i$. It is said $k$-step nilpotent if $\mathfrak{g}^{k}=0$ but $\mathfrak{g}^{k-1} \neq 0$. If $G$ is the unique simply connected nilpotent Lie group corresponding to a given nilpotent Lie algebra $\mathfrak{g}$, then the exponential map exp $: \mathfrak{g} \rightarrow G$ is a diffeomorphism [Ra].

Example 2.1. The Heisenberg Lie algebra is an example of a 2-step nilpotent Lie algebra, whose Lie group is called the Heisenberg Lie group $\mathbb{H}_{n}$. It can be constructed on $\mathbb{R}^{n} \times \mathbb{R}^{n} \times \mathbb{R}$ with the canonical topology and with the product

$$
\left(x, y, x_{0}\right)\left(x^{\prime}, y^{\prime}, x_{0}^{\prime}\right)=\left(x+x^{\prime}, y+y^{\prime}, x_{0}+x_{0}^{\prime}+\frac{1}{2}\left(x \cdot y^{\prime}-y \cdot x^{\prime}\right)\right),
$$

where $x, x^{\prime}, y, y^{\prime} \in \mathbb{R}^{n}$ and $x_{0}, x_{0}^{\prime} \in \mathbb{R}$. The identity element coincides with the origin $0=(0,0,0) . \mathbb{H}_{n}$ is non commutative and its center $\mathbb{Z}\left(\mathbb{H}^{n}\right)$ is the set of elements $\left(0,0, x_{0}\right)$ with $x_{0} \in \mathbb{R}$. Thus $\mathbb{H}^{n} / Z\left(\mathbb{H}^{n}\right) \simeq \mathbb{R}^{2 n}$ in the sense that every class is determined by the first two components $(x, y)$ and the product in the quotient group coincides with the sum on $\mathbb{R}^{2 n}$.

The left invariant vector fields at a point on $\mathbb{R}^{2 n+1}$ are

$$
X_{j}=\frac{\partial}{\partial x_{j}}-\frac{y_{j}}{2} \frac{\partial}{\partial x_{0}}, \quad Y_{j}=\frac{\partial}{\partial y_{j}}+\frac{x_{j}}{2} \frac{\partial}{\partial x_{0}}, \quad X_{0}=\frac{\partial}{\partial x_{0}} .
$$

It is easy to verify the Lie bracket relations:

$$
\left[X_{i}, Y_{j}\right]=\delta_{i j} X_{0} .
$$

Let $\mathfrak{h}_{n}$ be the Heisenberg Lie algebra of dimension $2 \mathrm{n}+1$, that is the Lie algebra of left invariant vector fields on $\mathbb{H}_{n}$, which coincides with $T_{0}\left(\mathbb{H}^{n}\right)$. Define a definite metric on $T\left(\mathbb{H}_{n}\right)$ so that the vectors $X_{i}, Y_{j}, X_{0}$ are orthonormal for all $\mathrm{i}, \mathrm{j}=1, \ldots, \mathrm{n}$. Then this metric is left invariant and can be transported to the quotient space $\mathbb{H}_{n} / Z\left(\mathbb{H}_{n}\right)$. The induced metric coincides with the canonical one on $\mathbb{R}^{2 n}$.

In the following section we shall see another construction of the Heisenberg Lie algebra with help of the Poisson bracket on $\mathbb{R}^{2 n}$.

Another class of Lie algebras (groups) is constitued by the solvable ones. Define ideals $D^{i}(\mathfrak{g})$ in $\mathfrak{g}$ by $D^{i}(\mathfrak{g})=\left[D^{i-1}(\mathfrak{g}), D^{i-1}(\mathfrak{g})\right]$ where $D^{0}(\mathfrak{g})=\mathfrak{g}$. The solvable Lie algebras are those for which there exists an integer $k$ such that $D^{k}(\mathfrak{g})=0$. In solvable Lie algebras the commutator $C(\mathfrak{g})=[\mathfrak{g}, \mathfrak{g}]$ is a nilpotent ideal.

Let $M$ be a smooth manifold and $\phi: G \times M \rightarrow M$ be a smooth action of $G$ on $M$. The vector fields on $M$

$$
\tilde{X}(m)=\left.\frac{d}{d t}\right|_{t=0} \phi(\exp t X, m) \quad m \in M, \quad X \in \mathfrak{g}, \quad t \in \mathbb{R}
$$

will denote the infinitesimal generators of this action. If $G \cdot m=\{\phi(g, m), g \in G\}$ denotes the $G$-orbit through $m \in M$ its tangent space is the set

$$
T_{m}(G \cdot m)=\{\tilde{X}(m) / X \in \mathfrak{g}\} .
$$

Here we also make use of the notation $g \cdot m=\phi(g, m)$. The following actions are important in our setting:

- the adjoint action Ad $: G \times \mathfrak{g} \rightarrow \mathfrak{g}$ whose infinitesimal generators are $\tilde{X}=\operatorname{ad}_{X}$, where $\operatorname{ad}_{X} Y=[X, Y]$ denotes the Lie bracket of $X, Y \in \mathfrak{g}$; 
- the coadjoint action of $G$ on $\mathfrak{g}^{*}$ is the dual of the adjoint action and it is given by $g \rightarrow \operatorname{Ad}^{*}\left(g^{-1}\right)$, for $g \in G$, whose infinitesimal generator is $\tilde{X}=-\operatorname{ad}_{X}^{*}$.

The coadjoint orbits are examples of symplectic manifolds. Recall that they are endowed with the Kirillov-Kostant-Souriau symplectic structure given by:

$$
\omega_{\beta}(\tilde{X}, \tilde{Y})=-\beta([X, Y]), \quad \beta \in G \cdot \mu .
$$

Assume now that $\mathfrak{g}$ has an ad-invariant metric $\langle\rangle:, \mathfrak{g} \times \mathfrak{g} \rightarrow \mathbb{R} ;$, that is, $\langle$,$\rangle is non-$ degenerate symmetric bilinear form for which the adjoint representation is skew symmetric. This gives rise to a bi-invariant pseudo Riemannian metric on the Lie group $G$ with Lie algebra $\mathfrak{g}$; bi-invariant means that the maps $\operatorname{Ad}(g)$ are isometries for all $g \in G$. Then $\langle$, induces a diffeomorphism between the adjoint orbit $G \cdot X$ and the coadjoint orbit $G \cdot \ell_{X}$ where $\ell_{X}(Y)=\langle X, Y\rangle$.

Recall that given a metric $\langle$,$\rangle on \mathfrak{g}$ the gradient of a function $f: \mathfrak{g} \rightarrow \mathbb{R}$ at the vector $X \in \mathfrak{g}$ is defined by

$$
\langle\nabla f(X), Y\rangle=d f_{X}(Y) \quad y \in \mathfrak{g} .
$$

Suppose that the Lie algebra $\mathfrak{g}$ admits a splitting

$$
\mathfrak{g}=\mathfrak{g}_{+} \oplus \mathfrak{g}_{-}
$$

as a direct sum of linear subspaces, where $\mathfrak{g}_{+}, \mathfrak{g}_{-}$are subalgebras of $\mathfrak{g}$. Then the Lie algebra $\mathfrak{g}$ also splits as $\mathfrak{g}=\mathfrak{g}_{+}^{\perp} \oplus \mathfrak{g}_{-}^{\perp}$, where $\mathfrak{g}_{ \pm}^{\perp}$ is isomorphic as vector spaces (via $\langle$,$\rangle ) to$ $\mathfrak{g}_{\mp}^{*}$. Let $G_{-}$denote a subgroup of $G$ with Lie algebra $\mathfrak{g}_{-}$. Then the coadjoint action of $G_{-}$on $\mathfrak{g}_{-}^{*}$ induces an action of $G_{-}$on $\mathfrak{g}_{+}^{\perp}$ :

$$
g_{-} \cdot X=\pi_{\mathfrak{g}_{+}^{\perp}}\left(\operatorname{Ad}\left(g_{-}\right) X\right) \quad g_{-} \in G_{-}, \quad X \in \mathfrak{g}_{+}^{\perp},
$$

where $\pi_{\mathfrak{g}_{+}^{\perp}}$ denotes the projection of $\mathfrak{g}$ on $\mathfrak{g}_{+}^{\perp}$. Thus the infinitesimal generator corresponding to $X_{-} \in \mathfrak{g}_{-}$is

$$
\tilde{X}_{-}(Y)=\pi_{\mathfrak{g}_{+}^{\perp}}\left(\left[X_{-}, Y\right]\right) \quad Y \in \mathfrak{g}_{+}^{\perp} .
$$

The orbit $G_{-} \cdot Y$ becomes a symplectic manifold with the symplectic structure given by

$$
\omega_{X}\left(\tilde{U}_{-}, \tilde{V}_{-}\right)=\left\langle X,\left[U_{-}, V_{-}\right]\right\rangle \quad \text { for } U_{-}, V_{-} \in \mathfrak{g}_{-}, X \in G_{-} \cdot Y
$$

which is induced by the Kostant-Kirillov-Souriau symplectic form on the coadjoint orbits in $\mathfrak{g}_{-}^{*}$.

Consider the restriction of the function $f: \mathfrak{g} \rightarrow \mathbb{R}$ to an orbit $G_{-} \cdot X:=\mathcal{M} \subset \mathfrak{g}_{+}^{\perp}$. Then the Hamiltonian vector field of $H=f_{\left.\right|_{\mathcal{M}}}$ is the infinitesimal generator corresponding to $-\nabla f_{-}$, that is

$$
X_{H}(Y)=-\pi_{\mathfrak{g}_{+}^{\perp}}\left(\left[\nabla f_{-}(Y), Y\right]\right)
$$

where $Z_{ \pm}$denotes the projection of $Z \in \mathfrak{g}$ with respect to the decomposition $\mathfrak{g}=\mathfrak{g}_{+} \oplus \mathfrak{g}_{-}$. In fact for $Y \in \mathfrak{g}_{+}^{\perp}, V_{-} \in \mathfrak{g}_{-}$we have

$$
\begin{aligned}
\omega_{Y}\left(\tilde{V}_{-}, X_{H}\right) & =d H_{Y}\left(\tilde{V}_{-}\right)=\left\langle\nabla f(Y), \pi_{\mathfrak{g}_{+}^{\perp}}\left(\left[V_{-}, Y\right]\right)\right\rangle=\left\langle\nabla f_{-}(Y),\left[V_{-}, Y\right]\right\rangle \\
& =\left\langle Y,\left[\nabla f_{-}(Y), V_{-}\right]\right\rangle=\omega_{Y}\left(\nabla f_{-}(Y), \tilde{V}_{-}\right) .
\end{aligned}
$$

Since $\omega$ is non degenerate, one gets (44). Therefore the Hamiltonian equation for $x: \mathbb{R} \rightarrow \mathfrak{g}$ follows

$$
x^{\prime}(t)=-\pi_{\mathfrak{g}_{+}^{\perp}}\left(\left[\nabla f_{-}(x), x\right]\right) .
$$


In particular if $f$ is ad-invariant then $0=[\nabla f(Y), Y]=\left[\nabla f_{-}(Y), Y\right]+\left[\nabla f_{+}(Y), Y\right]$. Since the metric is ad-invariant $\left[\mathfrak{g}_{+}, \mathfrak{g}_{+}^{\perp}\right] \subset \mathfrak{g}_{+}^{\perp}$ and thus equation (5) takes the form

$$
x^{\prime}(t)=\left[\nabla f_{+}(x), x\right]=\left[x, \nabla f_{-}(x)\right],
$$

hence (5) becomes a Lax equation, that is, it can be written as $x^{\prime}=[P(x), x]$.

If we assume now that the multiplication map $G_{+} \times G_{-} \rightarrow G,\left(g_{+}, g_{-}\right) \rightarrow g_{+} g_{-}$, is a diffeomorphism, then the initial value problem

$$
\left\{\begin{aligned}
\frac{d x}{d t} & =\left[\nabla f_{+}(x), x\right] \\
x(0) & =x_{0}
\end{aligned}\right.
$$

can be solved by factorization. In fact if $\exp t \nabla f\left(x_{0}\right)=g_{+}(t) g_{-}(t)$, then $x(t)=\operatorname{Ad}\left(g_{+}(t)\right) x_{0}$ is the solution of (7).

REMARK. If the multiplication map $G_{+} \times G_{-} \rightarrow G$ is a bijection onto an open subset of $G$, then equation (5) has a local solution in an interval $(-\varepsilon, \varepsilon)$ for some $\varepsilon>0$.

Recall that the Poisson bracket on $C^{\infty}(\mathfrak{g})$ is given by

$$
\{f, h\}(X)=\langle X,[\nabla f(X), \nabla h(X)]\rangle
$$

which is the Poisson bracket associated to the symplectic form on the adjoint orbits (the structure is induced via the metric from the coadjoint orbits).

A first step in the construction of action angle variables is to search for functions which Poisson commute. The Adler-Kostant-Symes Theorem shows a way to get functions in involution on the orbits $\mathcal{M}$. We shall formulate it in its classical Lie algebra setting.

Theorem 2.2. Let $\mathfrak{g}$ be a Lie algebra with an ad-invariant metric $\langle$,$\rangle . Assume \mathfrak{g}_{-}, \mathfrak{g}_{+}$are Lie subalgebras such that $\mathfrak{g}=\mathfrak{g}_{-} \oplus \mathfrak{g}_{+}$as direct sum of vector subspaces. Then any pair of ad-invariant functions on $\mathfrak{g}$ Poisson commute on $\mathfrak{g}_{+}^{\perp}$ (resp. on $\left.\mathfrak{g}_{-}^{\perp}\right)$.

Sometimes the ad-invariant condition is too strong, so the following version of the previous Theorem given by Ratiu [R1] ask for a weaker condition.

Theorem 2.3. Let $\mathfrak{g}$ be a Lie algebra carrying an ad-invariant metric $\langle$,$\rangle . Assume it$ admits a splitting into a direct sum as vector spaces $\mathfrak{g}=\mathfrak{g}_{+} \oplus \mathfrak{g}_{-}$, where $\mathfrak{g}_{+}$is an ideal and $\mathfrak{g}_{-}$is is a Lie subalgebra. If $f, h$ are smooth Poisson commuting functions on $\mathfrak{g}$, then the restrictions of $f$ and $h$ to $\mathfrak{g}_{+}^{\perp}$ are in involution in the Poisson structure of $\mathfrak{g}_{+}^{\perp}$.

Remark 2.4. This theorem was used in R2 to prove the involution of the Manakov integrals for the free n-dimensional rigid body motion.

2.1. The motion of $\mathbf{n}$ Harmonic oscillators. We shall apply the algebraic scheme of the previous section to the motion of $n$ uncoupled harmonic oscillators. This will be done with a solvable Lie algebra $\mathfrak{g}$ known as a oscillator Lie algebra, which can be endowed with an ad-invariant metric. This Lie algebra admits a splitting into a direct sum as vector spaces of two subalgebras. One of them is the Heisenberg Lie algebra, whose corresponding Lie group acts on the coadjoint orbits, included on $\mathfrak{g}$ via the ad-invariant metric on $\mathfrak{g}$. We choose a certain function and we realize the corresponding Hamiltonian system on the coadjoint orbits. The system is clearly equivalent to the linear one that approximates the motion of $\mathrm{n}$ uncoupled harmonic oscillators. Moreover we can show the complete integrability of the Hamiltonian on all maximal orbits.

The motion of $\mathrm{n}$ uncoupled harmonic oscillators can be approximated by a quadratic Hamiltonian $H$ as in (11) with $A=I d$, that is

$$
H=\frac{1}{2} \sum_{i}\left(p_{i}^{2}+q_{i}^{2}\right)
$$


where $q_{i}$ denote the position coordinates and $p_{i}=\dot{q}_{i}$ are the canonical momentum coordinates. Then (2) yields the following equation of motion

$$
\begin{aligned}
\frac{d q_{i}}{d t} & =p_{i} \\
\frac{d p_{i}}{d t} & =-q_{i}
\end{aligned}
$$

These equations predict the position and the velocity at any time if initial conditions $q_{i}\left(t_{0}\right), p_{i}\left(t_{0}\right)=\dot{q}_{i}\left(t_{0}\right)$ are known. The phase space in this case is $\mathbb{R}^{2 n}$, which is a symplectic manifold with the canonical structure given by

$$
\omega=\sum_{i} d q_{i} \wedge d p_{i}
$$

This has an associated Poisson structure, which for smooth functions $f, g$ on $\mathbb{R}^{2 n}$ is defined by

$$
\{f, g\}=(\nabla f, J \nabla g)=\sum_{i} \frac{\partial f}{\partial q_{i}} \frac{\partial g}{\partial p_{i}}-\frac{\partial f}{\partial p_{i}} \frac{\partial g}{\partial q_{i}} .
$$

With respect to this Lie bracket $\{$,$\} the subspace over \mathbb{R}$ generated by the functions $H=$ $\frac{1}{2} \sum_{i}\left(q_{i}^{2}+p_{i}^{2}\right)$, the coordinates $q_{i}, p_{i}$, and 1 form a solvable Lie algebra of dimension $2 \mathrm{n}+2$, which is a semidirect extension of the Heisenberg Lie algebra spanned by the functions $q_{i}, p_{i}, 1 \mathrm{i}=1, \ldots, \mathrm{n}$. In fact they obey the following non trivial rules

$$
\left\{q_{i}, p_{i}\right\}=1 \quad\left\{H, q_{i}\right\}=-p_{i} \quad\left\{H, p_{i}\right\}=q_{i} .
$$

In order to simplify notations let us rename these elements identifying $X_{n+1}$ with $H, X_{i}$ with $q_{i}, Y_{i}$ with $p_{i}$ and $X_{0}$ with the constant function 1 and set $\mathfrak{g}$ denotes the Lie algebra generated by these vectors with the Lie bracket [,] derived from the Poisson structure. This Lie algebra is known as a oscillator Lie algebra.

Consider the splitting of $\mathfrak{g}$ into a vector space direct sum $\mathfrak{g}=\mathfrak{g}_{+} \oplus \mathfrak{g}_{-}$, where $\mathfrak{g}_{ \pm}$denote the Lie subalgebras

$$
\mathfrak{g}_{-}=\operatorname{span}\left\{X_{0}, X_{i}, Y_{j}\right\}_{i, j=1, \ldots n}, \quad \mathfrak{g}_{+}=\mathbb{R} X_{n+1} .
$$

Notice that $\mathfrak{g}_{-}$is isomorphic to the $2 \mathrm{n}+1$-dimensional Heisenberg Lie algebra we denote $\mathfrak{h}_{n}$.

The quadratic form on $\mathfrak{g}$ which for $X=x_{0}(X) X_{0}+\sum_{i}\left(x_{i}(X) X_{i}+y_{i}(X) Y_{i}\right)+x_{n+1}(X) X_{n+1}$ is given by

$$
f(X)=\frac{1}{2} \sum_{i}\left(x_{i}^{2}+y_{i}^{2}\right)+x_{0} x_{n+1}
$$

induces an ad-invariant metric on $\mathfrak{g}$ denoted by $\langle$,$\rangle . Canonical computations show that$ the gradient of $f$ at a point $X$ is

$$
\nabla f(X)=X .
$$

The restriction of the quadratic form to $\mathfrak{v}:=\operatorname{span}\left\{X_{i}, Y_{j}\right\} \mathrm{i}, \mathrm{j}=1, \ldots, \mathrm{n}$, coincides with the canonical one on $\mathbb{R}^{2 n} \simeq \mathfrak{v}$. In other words the Lie algebra $\mathfrak{g}$ is the double extension of $\mathbb{R}^{2 n}$ with the canonical metric by the skew symmetric linear map which acts on $\mathfrak{v}$ as the restriction of $\operatorname{ad}\left(X_{n+1}\right)$ to this space (see for instance [M-R for the double extension procedure).

The metric induces a decomposition of the Lie algebra $\mathfrak{g}$ into a vector subspace direct sum of $\mathfrak{g}_{+}^{\perp}$ and $\mathfrak{g}_{-}^{\perp}$ where

$$
\mathfrak{g}_{-}^{\perp}=\operatorname{span}\left\{X_{0}\right\} \quad \mathfrak{g}_{+}^{\perp}=\mathbb{R} X_{n+1} \oplus \operatorname{span}\left\{X_{i}, Y_{j}\right\}_{i, j=1, \ldots, n},
$$


and it also induces linear isomorphisms $\mathfrak{g}_{ \pm}^{*} \simeq \mathfrak{g}_{\mp}^{\perp}$. Let $G$ denote a Lie group with Lie algebra $\mathfrak{g}$ and $G_{ \pm} \subset G$ is a Lie subgroup whose Lie algebra is $\mathfrak{g}_{ \pm}$. Hence the Lie subgroup $G_{-}$acts on $\mathfrak{g}_{+}^{\perp}$ by the "coadjoint" representation; indeed in terms of $U_{-} \in \mathfrak{g}_{-}$and $V \in \mathfrak{g}_{+}^{\perp}$ the infinitesimal action of $\mathfrak{g}_{-}$on $\mathfrak{g}_{+}^{\perp}$ is

$$
\operatorname{ad}_{U_{-}}^{*} V=x_{n+1}(V) \sum_{i}\left(y_{i}(U) X_{i}-x_{i}(U) Y_{i}\right)
$$

It is not difficult to see that the orbits are 2n-dimensional if $x_{n+1}(V) \neq 0$ and furthermore $V$ and $W$ belong to the same orbit if and only if $x_{n+1}(V)=x_{n+1}(W)$, hence the orbits are parametrized by the $x_{n+1}$-coordinate; so we denote them by $\mathcal{M}_{x_{n+1}}$. They are topologically like $\mathbb{R}^{2 n}$. In fact $\mathcal{M}_{x_{n+1}}=G_{-} \cdot V \simeq \mathbb{H}_{n} / Z\left(\mathbb{H}_{n}\right)$, where $\mathbb{H}_{n}$ denotes the Heisenberg Lie group with center $Z\left(\mathbb{H}_{n}\right)$.

Equipp these coadjoint orbits with the canonical symplectic structure. That is

$\omega_{Y}\left(\tilde{U}_{-}, \tilde{V}_{-}\right)=\left\langle Y,\left[U_{-}, V_{-}\right]\right\rangle=x_{n+1}(Y) \sum_{i=1}^{n}\left(x_{i}\left(U_{-}\right) y_{i}\left(V_{-}\right)-x_{i}\left(V_{-}\right) y_{i}\left(U_{-}\right)\right) \quad U_{-}, V_{-} \in \mathfrak{g}_{-}$.

Indeed on the orbit $\mathcal{M}_{1}$ the coordinates $x_{i}, y_{j}, i, j=1, \ldots n$, are the canonical symplectic coordinates and one can identify this orbit with $\mathbb{R}^{2 n}$ in a natural way.

Consider $H$, the restriction to a orbit $\mathcal{M}_{x_{n+1}}$ of the function $f$. Since $f$ is ad-invariant the Hamiltonian system of $H=f_{\left.\right|_{\mathcal{M}_{x_{n+1}}}}$ reduces to

$$
\begin{aligned}
\frac{d x}{d t} & =\left[x_{n+1} X_{n+1}, x_{\mathfrak{v}}+x_{n+1} X_{n+1}\right] \\
x(0) & =x^{0}
\end{aligned}
$$

where $x^{0}=x_{\mathfrak{v}}^{0}+x_{n+1}^{0} X_{0}$ and $x_{\mathfrak{v}}^{0}=\sum_{i}\left(x_{i}^{0} X_{i}+y_{i}^{0} Y_{i}\right)$.

For $x_{n+1} \equiv x_{n+1}^{0} \equiv 1$ this system is equivalent to (9).

The trajectories $x(t)$ with coordinates $x_{i}(t), y_{j}(t), x_{n+1}^{0}$ are parametrized circles of angular velocity $x_{n+1}^{0}$, for all $\mathrm{i}, \mathrm{j}$, that is

$$
\begin{aligned}
x_{i}(t) & =x_{i}^{0} \cos \left(x_{n+1}^{0} t\right)+y_{i}^{0} \sin \left(x_{n+1}^{0} t\right) \\
y_{j}(t) & =-x_{j}^{0} \sin \left(x_{n+1}^{0} t\right)+y_{j}^{0} \cos \left(x_{n+1}^{0} t\right) \\
x_{n+1}(t) & =x_{n+1}^{0}
\end{aligned}
$$

This solution coincides with that computed in the previous section, when we considered systems on coadjoint orbits. In fact it can be written as

$$
x(t)=\operatorname{Ad}\left(\exp t x_{n+1}^{0} X_{n+1}\right) x^{0},
$$

and one verifies that the flow at the point $X^{0} \in \mathfrak{g}_{+}^{\perp}$ is

$$
\begin{aligned}
\Delta^{t}\left(X^{0}\right)= & \sum_{i}\left[\left(x_{i}^{0} \cos \left(x_{n+1}^{0} t\right)+y_{i}^{0} \sin \left(x_{n+1}^{0} t\right)\right) X_{i}+\left(-x_{i}^{0} \sin \left(x_{n+1}^{0} t\right)+\right.\right. \\
& \left.\left.y_{i}^{0} \cos \left(x_{n+1}^{0} t\right)\right) Y_{i}\right]+x_{n+1}^{0} X_{n+1}
\end{aligned}
$$


System (13) is a Lax pair equation $L^{\prime}=[M, L]=M L-L M$, taking $L$ and $M$ the following matrices for $\omega_{i}=1$ for all i:

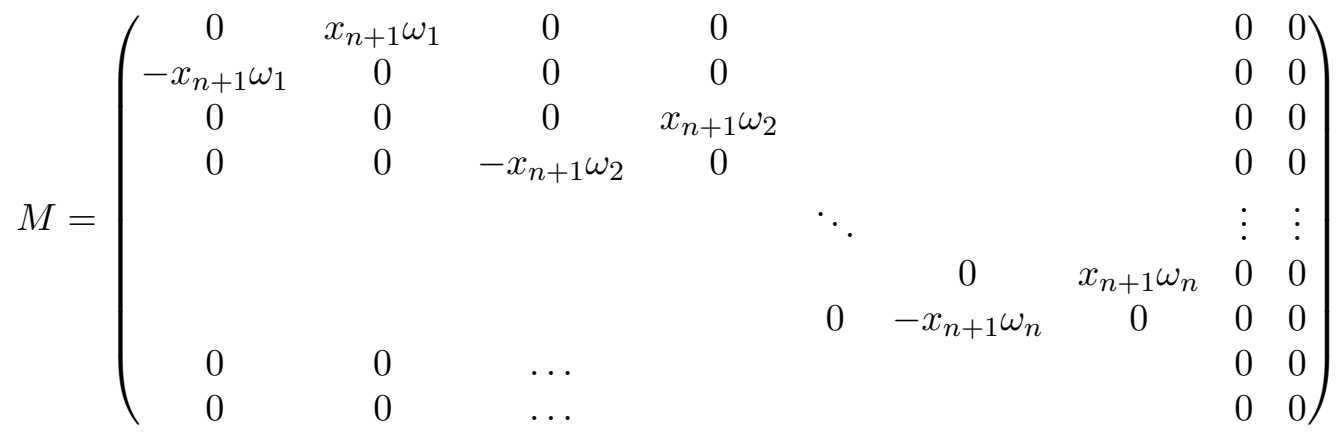

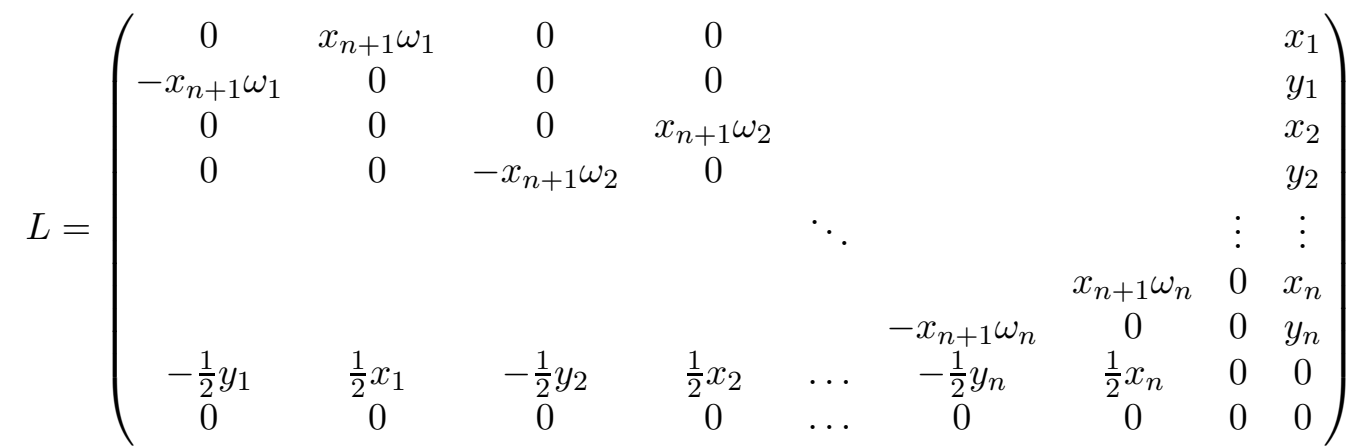

Next we shall prove the complete integrability of the function $H$, that is the restriction to the orbits $\mathcal{M}_{x_{n+1}}$ of the quadratic form associated to the ad-invariant metric on $\mathfrak{g}$. We shall show a set of $n$-functions that are in involution. However they are not Ad-invariant, hence they do not satisfy conditions of Theorem (2.2) and Theorem (2.3) does not hold in this case.

Definition 2.5. Recall that a function $f$ on a 2 n-dimensional Poisson manifold $(M,\{\}$, is completely integrable if there exist $n$ functions $f_{1}, \ldots, f_{n}$ such that:

i) $\left\{f, f_{i}\right\}=0,\left\{f_{i}, f_{j}\right\}=0$ for all $1 \leq i, j \leq n$,

ii) The differentials $d f_{1}, \ldots, d f_{n}$ are linearly independent on a open set invariant under the flow of $X_{f}$.

The Poisson structure on the orbits $\mathcal{M}_{x_{n+1}}$ is derived from the symplectic structure. Thus for a pair of functions $f, g: \mathfrak{g} \rightarrow \mathbb{R}$ the Poisson bracket of their respective restrictions $F, G$ at any point $X \in \mathfrak{g}_{+}^{\perp}$ is given by

$$
\{F, G\}(X)=\left\langle X,\left[\nabla f_{-}(X), \nabla g_{-}(X)\right]\right\rangle .
$$

Consider $H_{i}=f_{\left.i\right|_{\mathcal{M}_{n+1}}}$ be the restrictions to a orbit $\mathcal{M}_{x_{n+1}}$ of the functions

$$
f_{i}(X)=\frac{1}{2}\left(x_{i}^{2}+y_{i}^{2}\right)+x_{0} x_{n+1} \quad \text { for } i=1, \ldots n
$$

The functions $f_{i}$ are not ad-invariant but their restrictions commute with respect to the Poisson bracket induced by the Lie-Kirillov-Kostant symplectic form on the orbit. Moreover we assert

Proposition 2.6. The function $H$ is completely integrable on the orbits $\mathcal{M}_{x_{n+1}}$ for all $x_{n+1} \neq 0$. 
Proof. To prove this we first need to compute the gradient of $f_{i}$ which is $\nabla f_{i}(X)=$ $x_{i} X_{i}+y_{i} Y_{i}+x_{0} X_{0}+x_{n+1} X_{n+1}$.

Since $\left[\nabla f_{i}(X), \nabla f_{j}(X)\right]=0$ for all $\mathrm{i}, \mathrm{j}$, then $H_{i}, H_{j}$ Poisson commute on the orbits. In fact for $X \in \mathfrak{g}_{+}^{\perp}$

$$
\left\{H_{i}, H_{j}\right\}(X)=\left\langle X,\left[\nabla f_{i_{-}}(X), \nabla f_{j_{-}}(X)\right]\right\rangle=0
$$

where $V_{-}$denotes the projection of $V \in \mathfrak{g}$ with respect to the splitting $\mathfrak{g}=\mathfrak{g}_{+} \oplus \mathfrak{g}_{-}$. One can verify that the differentials $d f_{i}$ are linearly independent at any point and one proves the Poisson commutation with $H,\left\{H, H_{i}\right\}=0$ for all $i$.

The set

$$
\begin{aligned}
\mathcal{N}_{x_{n+1}} & =\cap_{i=1}^{n}\left\{X \in \mathcal{M}_{x_{n+1}}: H_{i}(X)=c_{i}\right\} \\
& =\cap_{i=1}^{n}\left\{X=\sum_{i}\left(x_{i} X_{i}+y_{i} Y_{i}\right)+x_{n+1} X_{n+1} \in \mathcal{M}_{x_{n+1}}: x_{i}^{2}+y_{i}^{2}=c_{i}\right\}
\end{aligned}
$$

is compact and nonempty for $c_{i} \geq 0$ for all i. Thus the Liouville Theorem applies and so it is possible to construct action angle coordinates, which can be written as $\left(H_{1}, \ldots, H_{n}, \theta_{1}, \ldots, \theta_{n}\right)$ where $\theta_{i}$ are the angle variables on the torus $\mathcal{N}_{x_{n+1}}$. In particular the flow $X_{H}$ in coordinates $\left(H_{1}, \ldots, H_{n}, \theta_{1}, \ldots, \theta_{n}\right)$ is linear.

Remark 2.7. Let $g: \mathfrak{g} \rightarrow \mathbb{R}$ be the quadratic polynomial of $\mathfrak{g}$ given by $g(X)=$ $\left\langle A X_{\mathfrak{v}}, X_{\mathfrak{v}}\right\rangle_{\mathfrak{v}}$, where $A$ is symmetric with respect to $\langle,\rangle_{\mathfrak{v}}$. Simple computations show that the gradient of $g$ has the form $\nabla g(X)=A X_{\mathfrak{v}}$ for $X \in \mathfrak{g}$. Let $H=g_{\left.\right|_{\mathcal{M}_{x_{n}+1}}}$ be the restriction of $g$ to the orbit, then following (15) the Hamiltonian system for $H$ is $x^{\prime}=\left[x_{+}, A x_{\mathfrak{v}}\right]=$

$x_{n+1} \operatorname{ad}_{X_{n+1}} A x_{\mathfrak{v}}=x_{n+1} J A x_{\mathfrak{v}}$ where $x$ is a curve on a orbit $x(t) \subset \mathcal{M}_{x_{n+1}} \subset \mathfrak{g}_{+}^{\perp}$ for all t. This Hamiltonian system is not a Lax pair equation. In the following section we shall consider a realization of this system like (2) as a Lax pair equation.

\section{Quadratic Hamiltonians And COADJoint orbits}

In this section we shall prove that Hamiltonian systems corresponding to quadratic Hamiltonians in $\mathbb{R}^{2 n}$ of the form $H(x)=\frac{1}{2}(A x, x)$ where $A$ is a symmetric map, can be described using the scheme of the previous section on a solvable Lie algebra. First we shall study the construction of solvable Lie algebras admitting an ad-invariant metric. We prove that quadratic Hamiltonians in $\mathbb{R}^{2 n}$ of the form (1) can be extended to a quadratic function on a solvable Lie algebra. This is the quadratic corresponding to an ad-invariant metric on the Lie algebra. These Lie algebras are semidirect extensions of the Heinsenberg Lie algebra. Secondly we shall equipp these solvable Lie algebras with an ad-invariant metric, on which we include the coadjoint orbits of the Heisenberg Lie group. On these orbits we realize the Hamiltonian systems corresponding to the quadratic of the ad-invariant metric. In this way the Hamiltonian system becomes a Lax equation, whose solution can be computed with help of the adjoint map in the solvable Lie group. Finally we discuss involution conditions for a class of functions on these codajoint orbits.

3.1. The structure of solvable Lie algebras admitting an ad-invariant metric. Let $\mathfrak{g}$ denote a Lie algebra endowed with an ad-invariant metric $\langle$,$\rangle , that is, \langle\rangle:, \mathfrak{g} \times \mathfrak{g} \rightarrow \mathbb{R}$ is a non degenerate symmetric bilinear form satisfying

$$
\langle[x, y], z\rangle+\langle y,[x, z]\rangle=0 \quad \text { for all } x, y, z \in \mathfrak{g} .
$$

Examples of real Lie algebras admitting an ad-invariant metric are the semisimple ones equipped with its Killing form $B: B(x, y)=\operatorname{tr}\left(\operatorname{ad}_{x} \operatorname{ad}_{y}\right)$ where $\operatorname{tr}$ denotes the trace and ad $: \mathfrak{g} \rightarrow \operatorname{End}(\mathfrak{g})$ is the adjoint representation.

If $G$ is a connected Lie group with Lie algebra $\mathfrak{g}$ then the pseudo Riemannian metric on $G$ obtained by left tranlations is also right invariant, or equivalently Ad-invariant. 
Lie algebras provided with an ad-invariant metric can be obtained in the following way. Let $(\mathfrak{b}, \varphi)$ be a orthogonal Lie algebra and let $S$ be a skew symmetric derivation of $(\mathfrak{b}, \varphi)$. Consider the vector space direct sum $\mathbb{R} Z \oplus \mathfrak{b} \oplus \mathbb{R} T$ and equipp this vector space with the following Lie bracket:

$$
\left[z_{1} Z+B_{1}+t_{1} T, z_{2} Z+B_{2}+t_{2} T\right]=\varphi\left(S B_{1}, B_{2}\right) Z+\left[B_{1}, B_{2}\right]_{\mathfrak{b}}+t_{1} S B_{2}-t_{2} S B_{1}
$$

where $z_{i}, t_{i} \in \mathbb{R}, i=1,2, B_{1}, B_{2} \in \mathfrak{b}$. The metric $\langle$,$\rangle on \mathfrak{g}=\mathbb{R} Z \oplus \mathfrak{b} \oplus \mathbb{R} T$ obtained as a orthogonal extension of $\varphi$, that is given by $\langle,\rangle_{\mathfrak{b} \times \mathfrak{b}}=\varphi$ and $\langle Z, T\rangle=1$ allows to extend $\varphi$ to an ad-invariant metric on $\mathfrak{g}$. The Lie algebra $(\mathfrak{g},\langle\rangle$,$) is called the double extension of$ $(\mathfrak{b}, \varphi)$ by $(\mathbb{R}, S)$.

It can be proved that any solvable Lie algebra $\mathfrak{g}$ endowed with an ad-invariant metric $\langle$,$\rangle is a double extension of a solvable Lie algebra with an ad-invariant metric (\mathfrak{b}, \varphi)$ by $\mathbb{R}$ with a certain skew symmetric derivation $S$ (see [M-R for instance).

The first examples of this method to get solvable Lie algebras with ad-invariant metrics follow from $\mathbb{R}^{m}$ endowed with a non degenerate symmetric form $b$. This $b$ can be written as

$$
b(X, Y)=(A X, Y) \quad \text { being } A \text { symmetric with respect to }(,),
$$

where $($,$) is the canonical inner product on \mathbb{R}^{m}$. Moreover $b$ is non degenerate if and only if $A$ is non singular.

Assume that $m=2 n$. Then the quadratic form corresponding to $b$ takes the form (11). Let $S$ be a linear transformation on $\mathbb{R}^{2 n}$. It is skew symmetric with respect to $b$, that is $b(S X, Y)=-b(X, S Y)$ if and only if $(A S X, Y)=-(A X, S Y)$.

If we apply the double extension procedure to $\left(\mathbb{R}^{2 n}, b\right)$ by $S$ we get the solvable Lie algebra $\mathfrak{g}=\mathbb{R} Z \oplus \mathbb{R}^{2 n} \oplus \mathbb{R} T$ with the Lie bracket

$$
\left[z_{1} Z+B_{1}+t_{1} T, z_{2} Z+B_{2}+t_{2} T\right]=\left(A S B_{1}, B_{2}\right) Z+t_{1} S B_{2}-t_{2} S B_{1} .
$$

Clearly $\mathbb{R} Z \oplus \mathbb{R}^{2 n}$ is a nilpotent ideal with a one dimensional commutator, hence $\mathfrak{g}$ is a semidirect extension of a Heisenberg Lie algebra $\mathfrak{h}_{m / 2}$. Furthermore $m / 2=n$ if and only if $A S$ is non singular. This says that if $A$ is injective, any non singular skew symmetric map for $b$ gives rise to a derivation of $\mathfrak{h}_{n}$ acting trivially on the center.

We shall prove a correspondence between the symmetric maps $A$ in $\mathbb{R}^{2 n}$ and the derivations of the Heisenberg Lie algebra acting trivially on the center.

If we fix the inner product on $\mathfrak{h}_{n}$ defined in Example (2.1) (denoted $\langle,\rangle^{\prime}$ ) then the Lie bracket on $\mathfrak{h}_{n}=\mathbb{R} X_{0} \oplus \mathfrak{v}$ where $\mathbb{R}^{2 n} \simeq \mathfrak{v}=\operatorname{span}\left\{X_{i}, Y_{j}\right\}_{i, j=1, \ldots, n}$ can be expressed as

$$
\left\langle[X, Y], x_{0} X_{0}\right\rangle^{\prime}=x_{0}\langle J X, Y\rangle^{\prime} \quad \text { with } J \text { as in (2) }
$$

and notice that $\langle,\rangle_{\left.\right|_{\mathfrak{v} \times \mathfrak{v}}}^{\prime}=($,$) . A derivation D$ of $\mathfrak{h}_{n}$ acting trivially on the center must satisfy $[D U, V]=-[U, D V]$ for all $U, V \in \mathfrak{v}$. Equivalently in terms of $\langle,\rangle^{\prime}$, we have that a map $D$ in $\mathfrak{h}_{n}$ is a derivation acting trivially on the center of $\mathfrak{h}_{n}$ if and only if the restriction of $D$ to $\mathfrak{v}$ (denoted also $D$ ) satisfies

$$
(J D U, V)=-(J U, D V) \quad \text { for all } U, V \in \mathfrak{v},
$$

where we replaced $\langle,\rangle_{\mathfrak{v}}^{\prime}$ by $($,$) since they coincide on \mathfrak{v} \simeq \mathbb{R}^{2 n}$. Denote by $\mathfrak{d}$ the set of derivations on $\mathfrak{h}_{n}$ acting trivially on the center of $\mathfrak{h}_{n}$.

Theorem 3.1. There is a bijection between the set of derivations of $\mathfrak{h}_{n}$ acting trivially on the center and the set $\mathfrak{s}$ of symmetric linear maps on $\mathbb{R}^{2 n}$. Explicitely this correspondence is given by the linear isomorphism $\psi$, which applies $D \in \mathfrak{d} \rightarrow J D \in \mathfrak{s}$, where $J$ is the complex structure as in (2). 
Moreover one can see that if $A$ is a symmetric map on $\mathbb{R}^{2 n}$ then $J A$ is always a skew symmetric map with respect to the bilinear map defined by $b(X, Y)=(A X, Y)$.

Proof. Let $D$ be an element of $\mathfrak{d}$, then $J D$ is symmetric with respect to the canonical inner product on $\mathbb{R}^{2 n}$. In fact $(J D U, V)=-(J U, D V)$ since $D$ is a derivation of $\mathfrak{h}_{n}$ and the assertion follows since $J$ is skew symmetric for $($,$) . For the converse let A$ be a symmetric transformation relative to $($,$) . Define a map D$ on $\mathfrak{h}_{n}$ by $D=J A$ on $\mathfrak{v}$ and extend it trivially to the center. It is easy to see that $D$ is a derivation of $\mathfrak{h}_{n}$.

To prove the second assertion let $A$ be a symmetric map on $\mathbb{R}^{2 n}$. Then we have $b(J A X, Y)=(A J A X, Y)=-(A X, J A Y)=-b(X, J A Y)$.

Thus for any non singular derivation $D$ of $\mathfrak{d}$ there always exists a symmetric non degenerate bilinear form on $\mathbb{R}^{2 n}$ with respect to which $D$ is skew symmetric. This is given by $b(X, Y)=(J D X, Y)$.

In the following section we shall apply this result to realize quadratic Hamiltonians on $\mathbb{R}^{2 n}$ on coadjoint orbits of the Heisenberg Lie group.

3.2. Quadratic Hamiltonians on coadjoint orbits of the Heisenberg Lie group. In this section we shall realize quadratic Hamiltonians of $\mathbb{R}^{2 n}$ (as in (10) on coadjoint orbits of the Heisenberg Lie group, which are included in a solvable Lie algebra admitting an ad-invariant metric. The corresponding Hamiltonian systems can be written as a Lax pair equation and the solution can be computed with help of the Adjoint map. Finally we shall prove involution conditions for a class of functions on these coadjoint orbits in terms of commutativity on the Lie algebra of derivations of $\mathfrak{h}_{n}$.

Consider a linear system of one degree of freedom on $\mathbb{R}^{2 n}$ with Hamiltonian given by:

$$
H(x)=\frac{1}{2}(A x, x)
$$

where $x=\left(q_{1}, \ldots, q_{n}, p_{1}, \ldots, p_{n}\right)$ is a vector in $\mathbb{R}^{2 n}$ written in a symplectic basis and $A$ is a symmetric linear operator with respect to the canonical inner product $($,$) . This yields$ the following Hamiltonian equation

$$
\text { (2) } \quad x^{\prime}=J A x, \quad \text { with } J=\left(\begin{array}{cc}
0 & -I d \\
I d & 0
\end{array}\right)
$$

and being $I d$ the identity. The phase space for this system is $\mathbb{R}^{2 n}$.

We shall construct a solvable Lie algebra that admits an ad-invariant metric on which the system (2) can be realized as a Hamiltonian system on coadjoint orbits. Moreover it can be written as a Lax pair equation.

Assume that $A$ is non singular and let $b$ denote the bilinear form on $\mathbb{R}^{2 n}=\operatorname{span}\left\{X_{i}, Y_{j}\right\}_{i, j=1}^{n}$ given by $b(X, Y)=(A X, Y)$. According to the previous section, $J A$ is skew symmetric with respect to $b$, where $J$ is the canonical complex structure on $\mathbb{R}^{2 n}$ as above. Let $\mathfrak{g}$ denote the double extension of $\left(\mathbb{R}^{2 n}, b\right)$ by $(\mathbb{R}, J A)$, that is $\mathfrak{g}=\mathbb{R} X_{0} \oplus \mathfrak{v} \oplus \mathbb{R} X_{n+1}$ with $\mathfrak{v}=\mathbb{R}^{2 n}$, where the Lie bracket is given by the non trivial relations

$$
[U, V]=b(J A U, V) Z_{0} \quad\left[X_{n+1}, U\right]=J A U \quad \text { for all } U \in \mathfrak{v},
$$

which can be equipped with the ad-invariant metric defined by

$$
\left\langle x_{0}^{1} X_{0}+U^{1}+x_{n+1}^{1} X_{n+1}, x_{0}^{2} X_{0}+U^{2}+x_{n+1}^{2} X_{n+1}\right\rangle=b\left(U^{1}, U^{2}\right)+\left(x_{0}^{1} x_{n+1}^{2}+x_{0}^{2} x_{n+1}^{1}\right) .
$$

Thus if $\langle,\rangle_{\mathfrak{v}}$ denotes the restriction of the metric of $\mathfrak{g}$ to $\mathfrak{v}=\operatorname{span}\left\{X_{i}, Y_{j}\right\}_{i, j=1, \ldots, n}$, then it coincides with the non degenerate symmetric bilinear map $b$ of $\mathbb{R}^{2 n}$ and $\mathfrak{g}$ admits a orthogonal splitting $\mathfrak{g}=\mathfrak{v} \oplus \operatorname{span}\left\{X_{0}, X_{n+1}\right\}$. 
Let $\mathfrak{g}_{ \pm}$denote the Lie subalgebras

$$
\mathfrak{g}_{+}=\mathbb{R} X_{n+1}, \quad \mathfrak{g}_{-}=\mathbb{R} X_{0} \oplus \operatorname{span}\left\{X_{i}, Y_{i}\right\} .
$$

Clearly they allow the splitting of $\mathfrak{g}$ into a vector space direct sum $\mathfrak{g}=\mathfrak{g}_{+} \oplus \mathfrak{g}_{-}$, which via the ad-invariant metric induces the following decomposition $\mathfrak{g}=\mathfrak{g}_{+}^{\perp} \oplus \mathfrak{g}_{-}^{\perp}$, direct sum as vector spaces, where

$$
\mathfrak{g}_{-}^{\perp}=\mathbb{R}\left\{X_{0}\right\} \quad \mathfrak{g}_{+}^{\perp}=\operatorname{span}\left\{X_{i}, Y_{i}\right\}_{i=1, \ldots, n} \oplus \mathbb{R} X_{n+1} .
$$

Indeed $\mathfrak{g}_{-}$is an ideal of $\mathfrak{g}$ isomorphic to the $2 \mathrm{n}+1$-dimensional Heisenberg Lie algebra $\mathfrak{h}_{n}$.

If $G$ denotes a Lie group with Lie algebra $\mathfrak{g}$, set $G_{-} \subset G$ the Lie subgroup with Lie subalgebra $\mathfrak{g}_{-}$. Then $G_{-}$acts on $\mathfrak{g}_{+}^{\perp}$ by the coadjoint action

$$
g_{-} \cdot X=\pi_{\mathfrak{g}_{+}^{\perp}}\left(\operatorname{Ad}\left(g_{-}\right) X\right) \quad g_{-} \in G_{-}, \quad X \in \mathfrak{g}_{+}^{\perp},
$$

where $\pi_{\mathfrak{g}_{+}^{\perp}}$ denotes the projection of $\mathfrak{g}$ on $\mathfrak{g}_{+}^{\perp}$, which in infinitesimal terms gives the following action of $\mathfrak{g}_{-}$on $\mathfrak{g}_{+}^{\perp}$

$$
\operatorname{ad}_{U}^{*} V:=U \cdot V=x_{n+1}(V) J A X_{\mathfrak{v}}(U) \quad \text { for } U \in \mathfrak{g}_{-}, V \in \mathfrak{g}_{+}^{\perp} .
$$

where $X_{\mathfrak{v}}(U)$ denotes the projection of $U$ onto $\mathfrak{v}$ with respect to the orthogonal splitting $\mathfrak{g}=\left(\mathbb{R} X_{0} \oplus \mathbb{R} X_{n+1}\right) \oplus \mathfrak{v}$. The orbits are 2n-dimensional if $x_{n+1}(V) \neq 0$ and furthermore $V$ and $W$ belong to the same orbit if and only if $x_{n+1}(V)=x_{n+1}(W)$, and this allows to parametrize the orbits by the $x_{n+1}$-coordinate; so we denote them by $\mathcal{M}_{x_{n+1}}$. The orbits are topologically like $\mathbb{R}^{2 n}$ since they are diffeomorphic to the quotient $\mathbb{H}_{n} / Z\left(\mathbb{H}_{n}\right)$, where $Z\left(\mathbb{H}_{n}\right)=\mathbb{R} X_{0}$.

Endow the orbits with the canonical symplectic structure of the coadjoint orbits, that is

$$
\omega_{X}\left(\tilde{U}_{-}, \tilde{V}_{-}\right)=\left\langle X,\left[U_{-}, V_{-}\right]\right\rangle=x_{n+1}(X) b\left(J A U_{\mathfrak{v}}, V_{\mathfrak{v}}\right)
$$

for $X \in \mathfrak{g}_{+}^{\perp}, U_{-}, V_{-} \in \mathfrak{g}_{-}$.

Let $f: \mathfrak{g} \rightarrow \mathbb{R}$ be the ad-invariant function given by $f(X)=\frac{1}{2}\langle X, X\rangle$. The gradient of the function $f$ at a point $X$ is the position vector $\nabla f(X)=X$. Since $f$ is ad-invariant the Hamiltonian system of $H=f_{\left.\right|_{\mathcal{M}_{x_{n}+1}}}$, the restriction of $f$ to the orbit $\mathcal{M}_{x_{n+1}}$, is given by (6), so we have

$$
\begin{aligned}
\frac{d x}{d t} & =\left[\nabla f_{+}(x), x\right]=\left[x_{n+1} X_{n+1}, x_{\mathfrak{v}}+x_{n+1} X_{n+1}\right]=x_{n+1} J A x_{\mathfrak{v}} \\
x(0) & =X^{0}
\end{aligned}
$$

where $X^{0} \in \mathfrak{g}_{+}^{\perp}$. This Hamiltonian system written as a Lax pair equation is equivalent to (2) for $x_{n+1}=x_{n+1}^{0}=1$. The solution for the initial condition $X^{0} \in \mathfrak{g}_{+}^{\perp}$ can be computed with help of the Adjoint map on $G$. In fact it can be written as

$$
X(t)=\operatorname{Ad}\left(\exp t x_{n+1}^{0} X_{n+1}\right) X^{0} .
$$

The previous explanations prove the following result.

Theorem 3.2. Let $H(X)=\frac{1}{2}(A X, X)$ be a quadratic Hamiltonian on $\mathbb{R}^{2 n}$ with corresponding Hamiltonian system (2). Then $H$ can be extended to a quadratic function $f$ on a solvable Lie algebra $\mathfrak{g}$ containing the Heisenberg Lie algebra as a proper ideal. The function $f$ induces a Hamiltonian system on coadjoint orbits of the Heisenberg Lie group, that can be written as a Lax pair equation and which is equivalent to (2). Moreover the trajectories on $\mathbb{R}^{2 n}$ for the initial condition $V^{0}$ can be computed with help of the Adjoint map on $\mathfrak{g}$. Explicitely they are the curves $x(t)=\exp ^{t J A} V^{0}$, where exp denotes the usual exponential map of matrices. 
Remark 3.3. The Lie algebra $\mathfrak{g}$ above is isomorphic to the Lie algebra of real functions on $\mathbb{R}^{2 n}$ under Poisson bracket generated by $q_{i}, p_{j}, 1$ and the Hamiltonian $H$.

Example 3.4 (The motion of n-uncoupled inverse pendula). As example of the previous construction consider the linear approximation of the motion of $\mathrm{n}$ uncoupled inverse pendula. This corresponds to the Hamiltonian $H(x)=\frac{1}{2}(A x, x)$ where

$$
A=\left(\begin{array}{cc}
I d & 0 \\
0 & -I d
\end{array}\right)
$$

This yields the Hamiltonian system $x^{\prime}=J A x$, which in coordinates takes the form

$$
\begin{aligned}
\frac{d q_{i}}{d t} & =p_{i} \\
\frac{d p_{i}}{d t} & =q_{i}
\end{aligned}
$$

The phase space for this system is $\mathbb{R}^{2 n}$. By considering the setting above we construct coadjoint orbits $\mathcal{M}$ of the Heisenberg Lie group, that are included in a solvable Lie algebra $\mathfrak{g}$ with Lie bracket (17) and ad-invariant metric (18). The Hamilonian system for the restriction to the orbits of the ad-invariant function on $\mathfrak{g}$ induced by the metric can be written as

$$
\begin{aligned}
\frac{d x}{d t} & =\left[x_{n+1} X_{n+1}, x_{\mathfrak{v}}+x_{n+1} X_{n+1}\right] \\
x(0) & =X^{0}
\end{aligned}
$$

where $X^{0}=\sum_{i}\left(x_{i}^{0} X_{i}+y_{i}^{0} Y_{i}\right)+x_{n+1}^{0} X_{n+1}$. This is in fact a Lax pair equation. If we identify the coordinates $q_{i}$ with $x_{i}$ and $p_{i}$ with $y_{i}$ then the Hamiltonian system above on the coadjoint orbit $\mathcal{M}_{1}$ written in coordinates is clearly equivalent to (21).

The trajectories on $\mathfrak{g}_{+}^{\perp}, x=\sum_{i}\left(x_{i}(t) X_{i}+y_{i}(t) Y_{i}\right)+x_{n+1} X_{n+1}$ are parametrized by

$$
\begin{aligned}
x_{i}(t) & =x_{i}^{0} \cosh \left(x_{n+1}^{0} t\right)+y_{i}^{0} \sinh \left(x_{n+1}^{0} t\right) \\
y_{i}(t) & =x_{i}^{0} \sinh \left(x_{n+1}^{0} t\right)+y_{i}^{0} \cosh \left(x_{n+1}^{0} t\right) \\
x_{n+1}(t) & =x_{n+1}^{0}
\end{aligned}
$$

One can verify that the flow at the point $X^{0} \in \mathfrak{g}_{+}^{\perp}$ is then

$$
\begin{aligned}
\Delta^{t}\left(X^{0}\right)= & \sum_{i}\left[\left(x_{i}^{0} \cosh \left(x_{n+1}^{0} t\right)-y_{i}^{0} \sinh \left(x_{n+1}^{0} t\right) X_{i}+\right.\right. \\
& +\left(x_{i}^{0} \sinh \left(x_{n+1}^{0} t\right)+y_{i}^{0} \cosh \left(x_{n+1}^{0} t\right) Y_{i}\right]+x_{n+1}^{0} X_{n+1}
\end{aligned}
$$

System (22) is a Lax pair equation $L^{\prime}=[M, L]=M L-L M$, taking $L$ and $M$ the following matrices in $M(2 n+2, \mathbb{R})$ with $\omega_{i}=1$ :

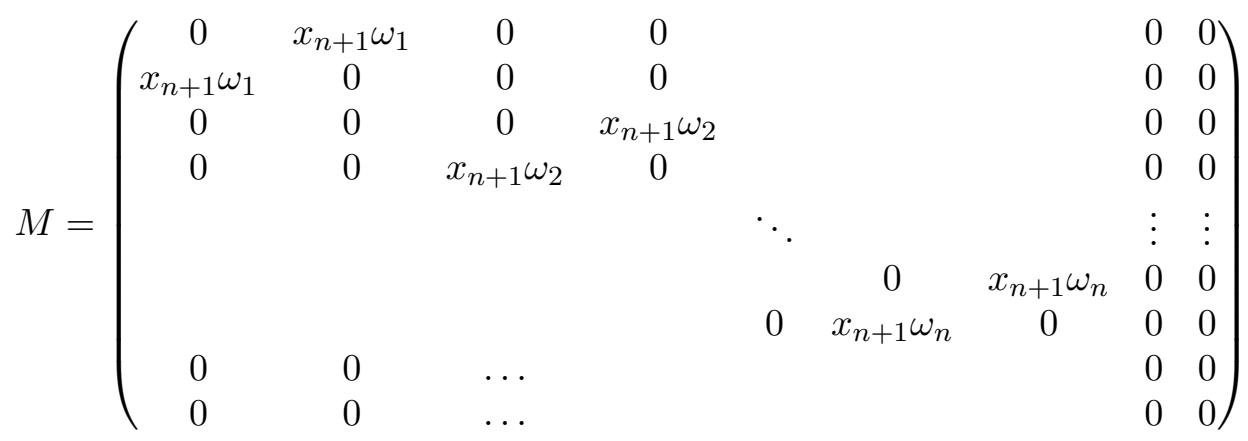




$$
L=\left(\begin{array}{ccccccccc}
0 & x_{n+1} \omega_{1} & 0 & 0 & & & & & x_{1} \\
x_{n+1} \omega_{1} & 0 & 0 & 0 & & & & \\
0 & 0 & 0 & x_{n+1} \omega_{2} & & & & y_{1} \\
0 & 0 & x_{n+1} \omega_{2} & 0 & & & & & y_{2} \\
& & & & \ddots & & & \vdots & \vdots \\
& & & & & & x_{n+1} \omega_{n} & 0 & x_{n} \\
& & & & & x_{n+1} \omega_{n} & 0 & 0 & y_{n} \\
-\frac{1}{2} y_{1} & \frac{1}{2} x_{1} & -\frac{1}{2} y_{2} & \frac{1}{2} x_{2} & \ldots & -\frac{1}{2} y_{n} & \frac{1}{2} x_{n} & 0 & 0 \\
0 & 0 & 0 & 0 & \ldots & 0 & 0 & 0 & 0
\end{array}\right)
$$

Next we shall prove the complete integrability (2.5) of the function $H$ with system (22). We shall show a set of n-functions that are in involution. As in the case of the n-uncoupled harmonic oscillators they are not Ad-invariant, hence they do not satisfy conditions of Theorem (2.2) and Theorem (2.3) does not hold in this case.

Consider $H_{i}=f_{i_{\mathcal{M}_{x_{n+1}}}}$ be the restrictions to a orbit $\mathcal{M}_{x_{n+1}}$ of the functions

$$
f_{i}(X)=\frac{1}{2}\left(y_{i}^{2}-x_{i}^{2}\right) \quad \text { for } i=1, \ldots n
$$

The functions $f_{i}$ are not ad-invariant but their restrictions Poisson commute on the orbit. Moreover we assert

Proposition 3.5. The function $H$ is completely integrable (in the sense of (2.5)) on the orbits $\mathcal{M}_{x_{n+1}}$ for all $x_{n+1} \neq 0$.

Proof. The proof follows from a analogous procedure as in (2.6).

However in this case the set

$$
\begin{aligned}
\mathcal{N}_{x_{n+1}} & =\cap_{i=1}^{n}\left\{X \in \mathcal{M}_{x_{n+1}}: H_{i}(X)=c_{i}\right\} \\
& =\cap_{i=1}^{n}\left\{X=\sum_{i}\left(x_{i} X_{i}+y_{i} Y_{i}\right)+x_{n+1} X_{n+1} \in \mathcal{M}_{x_{n+1}}: x_{i}^{2}+y_{i}^{2}=c_{i}\right\}
\end{aligned}
$$

is not compact.

Remark 3.6. If $\mathfrak{g}_{(4)}$ denotes the four dimensional Lie algebra of the example above, the connected Lie group $G=\exp \mathfrak{g}$ is called the Boidol group.

Motivated by the involution conditions proved in the equation of motion of both systems corresponding to n-uncoupled harmonic oscillators and n-uncoupled inverse pendula, we shall investigate involution conditions on the coadjoint orbits of the Heisenberg Lie group for the restrictions of the quadratic functions $f(X)=\frac{1}{2}\langle X, X\rangle$, where $\langle$,$\rangle denotes the$ ad-invariant metric on the solvable Lie algebra $\mathfrak{g}$.

Let $g_{i}, g_{j}$ be two quadratic on $\mathbb{R}^{2 n}$ associated to symmetric maps $A_{i}, A_{j}: \mathfrak{v} \rightarrow \mathfrak{v}$ respectively, that is

$$
g_{i}(X)=\frac{1}{2}\left(A_{i} X, X\right) \quad g_{j}(X)=\frac{1}{2}\left(A_{j} X, X\right) .
$$

Consider the quadratic functions on the solvable Lie algebra $\mathfrak{g}$, that are extensions of $g_{i}, g_{j}$ to $\mathbb{R} X_{0} \oplus \mathbb{R} X_{n+1}$, for instance as

$$
g_{i}(X)=\frac{1}{2}\left(A_{i} X_{\mathfrak{v}}, X_{\mathfrak{v}}\right)+x_{0} x_{n+1} \quad g_{j}(X)=\frac{1}{2}\left(A_{j} X_{\mathfrak{v}}, X_{\mathfrak{v}}\right)+x_{0} x_{n+1} .
$$

For the following results these extensions are not unique. For instance extending them trivially we get the same conclusions.

Let $H_{i}, H_{j}$ denote the restrictions of $g_{i}, g_{j}$ to the orbits $\mathcal{M}_{x_{n+1}}$ and let $X \in \mathcal{M}_{x_{n+1}} \subset \mathfrak{g}_{+}^{\perp}$. The Poisson bracket of the functions $H_{i}, H_{j}$ on the orbit follows:

$$
\left\{H_{i}, H_{j}\right\}(X)=\left\langle X,\left[\nabla g_{i_{-}}(X), \nabla g_{j_{-}}(X)\right]\right\rangle
$$


Thus we need to compute the gradients of $g_{i}, g_{j}$, which are

$$
\nabla g_{i}(X)=A^{-1} A_{i} X_{\mathfrak{v}}+x_{0} X_{0}+x_{n+1} X_{n+1} \quad \nabla g_{j}(X)=A^{-1} A_{j} X_{\mathfrak{v}}+x_{0} X_{0}+x_{n+1} X_{n+1} .
$$

In Theorem (3.1) we established a correspondence between the symmetric maps on $\mathbb{R}^{2 n}$ and the derivations of $\mathfrak{h}_{n}$ acting trivially on the center, given by $\psi$ which sends $A \rightarrow J A$, where $J$ is the canonical complex structure on $\mathfrak{v}$ and $J A$ is extended trivially to the center of $\mathfrak{h}_{n}$.

Theorem 3.7. The functions $H_{i}, H_{j}$ are in involution on the orbits $\mathcal{M}_{x_{n+1}}$ if and only if

$$
\left[\psi\left(A_{i}\right), \psi\left(A_{j}\right)\right]=0
$$

Proof. Let $X \in \mathcal{M}_{x_{n+1}} \subset \mathfrak{g}_{+}^{\perp}$. Then for the functions $H_{i}, H_{j}$ the Poisson bracket on the orbit follows:

$$
\begin{aligned}
\left\{H_{i}, H_{j}\right\}(X) & =\left\langle X,\left[A_{i} X_{\mathfrak{v}}, A_{j} X_{\mathfrak{v}}\right]\right\rangle=\left\langle x_{n+1}\left[X_{n+1}, A^{-1} A_{i} X_{\mathfrak{v}}\right], A^{-1} A_{j} X_{\mathfrak{v}}\right\rangle \\
& =x_{n+1}\left\langle J A_{i} X_{\mathfrak{v}}, A^{-1} A_{j} X_{\mathfrak{v}}\right\rangle=x_{n+1}\left(J A_{i} X_{\mathfrak{v}}, A_{j} X_{\mathfrak{v}}\right)
\end{aligned}
$$

Therefore $\left\{H_{i}, H_{j}\right\}(X)=0$ if and only if $\left(A_{j} J A_{i} X_{\mathfrak{v}}, X_{\mathfrak{v}}\right\rangle=0$ which is equivalent to $A_{j} J A_{i}=A_{i} J A_{j}$, if and only if $J A_{j} J A_{i}=J A_{i} J A_{j}$. Since $\psi(A)=J A$ we proved the result.

Corollary 3.8. If there exists an $n$-dimensional abelian subalgebra on $z(J A)_{\mathfrak{d}}$, where

$$
z(J A)_{\mathfrak{d}}=\{D \in \mathfrak{d} \text { such that }[D, J A]=0\}
$$

then the Hamiltonian function $H$ restriction of the function $f(X)=\frac{1}{2}(A X, X)$ is completely integrable on the orbits $\mathcal{M}_{x_{n+1}}$ for $x_{n+1} \neq 0$.

Proof. The previous theorem says that the restrictions to the orbit $\mathcal{M}_{x_{n+1}}$ of the functions $g_{i}, g_{j}$ are in involution if their corresponding derivations commute in $\mathfrak{d}$. In particular for $g_{i}$ and $f$, we have that $H$ and $H_{i}$ Poisson commute on the orbit if and only if $J A_{i}$ belongs to the centralizer of $J A$ in $\mathfrak{d}, z(J A)_{\mathfrak{d}}$. Since the complete integrability requires of $\mathrm{n}$ linearly independent functions, this can be done with a basis of a $n$-dimensional abelian subalgebra of $z(J A)_{\mathfrak{d}}$, finishing the proof.

Thus using Lie Theory we can say that many of these systems are completely integrable. In fact the Lie algebra of derivations of $\mathfrak{h}_{n}$ acting trivially on the center is $s p(n)$ (see [Sa]), which has a Cartan decomposition o the form $s p(n)=\mathfrak{u} \oplus \mathfrak{p}$, where $\mathfrak{u}$ is a maximal compact subalgebra. Taking for instance any element in $\mathfrak{u}$, then it induces a completely integrable system. More generally we should study the abelian subalgebras on $s p(n)$.

Example 3.9 (Involution on the oscillators). Note that whenever we choose $A=I d$ as the symmetric map for $H$ in (11), and we apply the double extension procedure we get the oscillator Lie algebra $\mathfrak{g}$ endowed with its Lorentzian ad-invariant metric. Let $H$ denote as above the restriction to the coadjoint orbits of the function induced by the metric. Reading the previous proposition in this situation we have that:

$\left\{H, H_{i}\right\}=0$ if and only if $\psi\left(A_{i}\right)$ belongs to the Lie subalgebra of isometries of $\mathbb{H}_{n}$ fixing the identity and acting trivially on the center.

In fact the Lie algebra of the isometries of $\mathbb{H}_{n}$ fixing the identity is the set of skew symmetric derivations of $\mathfrak{h}_{n}$, that is those derivations satisfying $\langle D u, v\rangle^{\prime}=-\langle u, D v\rangle^{\prime}$, where $\langle,\rangle^{\prime}$ is the canonical inner product on $\mathbb{H}_{n}$ as in (2.1). The previous results says that $H_{i}$ Poisson commutes with $H$ if and only if $A_{i} J=J A_{i}$. But $J A_{i}$ can be identified with a 
derivation $D$ of $\mathfrak{h}_{n}$ acting trivially on the center and $\left(J A_{i}\right)^{t}=-A_{i} J=-J A_{i}$, that is $D$ is skew symmetric, and this proves our assertion.

Explicitely, let $J: \mathfrak{v} \rightarrow \mathfrak{v}$ denote the canonical complex structure of $\mathbb{R}^{2 n}$ defined as (2) in the Introduction. Notice that by identifying $\mathbb{R}^{2 n}$ with $\mathfrak{v}$ as isometric vector spaces then $J$ coincides with the restriction of $\operatorname{ad}_{X_{n+1}}$ to $\mathfrak{v}$ in the oscillator Lie algebra $\mathfrak{g}$. The restriction to the orbit of a quadratic $g(X)=\langle A X, X\rangle$ with a symmetric map $A: \mathfrak{v} \rightarrow \mathfrak{v}$ of the form

$$
\left(\begin{array}{ll}
B & C \\
D & E
\end{array}\right)
$$

Poisson commutes with $H$ the restriction of the quadratic induced by the metric on $\mathfrak{g}$ if and only if $C=-D$ and $B=E$ where $B$ is also symmetric. That is, the matrix $A$ seen as a linear map on $\mathbb{R}^{2 n}$ is complex and symmetric. Assume now that the restrictions of two quadratic functions $g_{i}, g_{j}$ associated to symmetric maps $A_{i}, A_{j}$ are in involution with $H$. Then they pairwise Poisson commute if and only if $\left[C_{i}, B_{j}\right]=\left[C_{j}, B_{i}\right]$ and $\left[C_{i}, C_{j}\right]=$ $\left[B_{i}, B_{j}\right]$ for all $\mathrm{i}, \mathrm{j}$, where [, $]$ is the canonical Lie bracket for matrices: $[A, B]=A B-B A$.

In particular the functions $H_{i}$ defined as the restrictions to the orbit $\mathcal{M}_{x_{n+1}}$ of the functions $f_{i}(X)=\frac{1}{2}\left(x_{i}^{2}+y_{i}^{2}\right)+x_{0} x_{n+1}$ as in (15) are examples of the quadratic functions above.

Conclusions We studied Hamiltonian systems on coadjoint orbits of the Heisenberg Lie algebra. This was done with Lie theory as a powerful tool. The Heisenberg Lie algebra was included in a solvable Lie algebra admitting an ad-invariant metric. The metric was useful to write the corresponding Hamiltonian system in a Lax form. Next we study Poisson commutativity. The involution conditions for a class of functions are related to the Heisenberg Lie algebra. This work shows examples of applications of the AdlerKostant-Symes scheme on solvable Lie algebras. This allows to ask for new examples and a more general theory for Poisson commutativity.

\section{REFERENCES}

[AM] Abraham, R., Marsden, J, Foundations of Mechanics, Second edition. The Benjamin Cummings publishing company, (1985).

[A] AdLeR, M., On a trace for formal pseudodifferential operators and the symplectic structure for the KdV type equations, Invent. Math., 50, 219-248, (1979).

[Ar] Arnold, V. I., Mathematical methods of classical mechanics, Springer Verlag, (1980).

[B-K] Baum, H., Kath, I., Doubly extended Lie groups ' curvature, holonomy and parallel spinors, Diff. Geom. Appl., 19, 253-280, (1998).

[G] Guest, M, Harmonic Maps, Loop Groups and Integrable Systems. (London Math.Soc.Student Texts; 38). New York: Cambridge University Press (1997).

[G-S] Gulllemin, V., Sternberg, S., Symplectic tecniques in physics. Cambridge New York Port Chester Melbourne Sydney: Cambridge University Press (1991).

[F-M] Fomenko, A., Mischenko, A., Generalized Liouville method of integration of Hamiltonian systems, Funct. Anal. and its Applic., 12, 113 -121, (1978).

[Ko1] Kostant, B., Quantization and Representation Theory, in: Representation Theory of Lie groups, Proc. SRC/LMS Res. Symp., Oxford 197\%. London Math. Soc. Lecture Notes Series, 34, 287-316, (1979).

[Ko2] Kostant, B., The solution to a generalized Toda lattice and representation theory, Advances in Math., 39, 195 - 338, (1979).

[M] Medina, A., Groupes de Lie munis de pseudo-metriques de Riemann bi-invariantes, Sém. Géom. Diff., (1981-1982), Montepellier.

[M-R] Medina, A., Revoy, Ph., Algèbres de Lie et produit scalaire invariant, Ann. scient. Éc. Norm. Sup., $4^{e}$ série, t. 18, 391 - 404, (1985). 
[O1] Ovando, G., Estructuras complejas y sistemas hamiltonianos en grupos de Lie solubles, Tesis Doctoral, Fa.M.A.F. Univ. Nac. de Córdoba,( Marzo 2002).

[Ra] Raghunathan, M., Discrete subgroups of Lie groups, Springer, New York,(1972).

[R1] Ratiu, T., Involution theorems, Geometric methods in Math. Phys., Lect. Notes in Math., 775, Procedings, Lowell, Massachusetts 1979, Springer Verlag, (1980).

[R2] Ratiu, T., The motion of the free n-dimensional rigid body, Indiana Univ. Math. Journal, 29, $609-629,(1980)$.

[Sa] SAAL, L. The automorphism group of a Lie algebra of Heisenberg type Rend. Sem. Mat. Univ. Pol. Torino, 54 2, (1996).

[Sy] Symes, W., Systems of Toda type, inverse spectral problems and representation theory, Invent. Math., 59, 13 - 53, (1978).

[Va] Varadarajan, V., Lie groups, Lie algebras and their representations, Springer, (1984).

Ciem - Facultad de Matemática, Astronomía y Física, Universidad Nacional de Córdoba, Córdoba 5000, Argentina

E-mail address: ovando@mate.uncor.edu 